\title{
Routine diagnostic procedures for chronic encephalopathy induced by solvents: survey of experts
}

\author{
J A F van der Hoek, M M Verberk, G van der Laan, G Hageman
}

\begin{abstract}
Objectives-To study the routine diagnostic procedures used in different countries for chronic toxic encephalopathy (CTE) induced by solvents.

Methods-By means of a postal questionnaire selected international experts were asked about the methods they use to diagnose patients suspected of having CTE induced by solvents, the number of patients, entrance criteria, and the results of these diagnostic procedures.

Results-18 Experts working in 18 diagnostic centres responded. Most of them agreed that a diagnostic procedure for CTE induced by solvents should contain an interview and neurological, physical, and neuropsychological examinations. However, the tests used were very different, as were the classifications for CTE. Depending on the institute, a diagnosis of CTE was made in $6 \%-70 \%$ of the referred patients. The proportion of patients with CTE stage I ranged from $0 \%$ to $33 \%$, stage II from $5 \%$ to $100 \%$, and stage III from $0 \%$ to $95 \%$.
\end{abstract}

Conclusion-The intentions of the two 1985 conferences that aimed at clarity and uniformity of diagnosis of CTE induced by solvents are far from reached. It is possible, now the conditions are more favourable, to aim at this important goal and recommend some refinement of the then proposed criteria.

(Occup Environ Med 2001;58:382-385)

Occupational

Diseases, Coronel

Institute, Academic

Medical Center,

University of

Amsterdam,

Amsterdam, The

Netherlands

J A F van der Hoek

$M$ M Verberk

$\mathrm{G}$ van der Laan

Department of

Neurology, Medical

Spectrum Twente, PO

Box 50000, NL-7500 KA

Enschede, The

Netherlands

J A F van der Hoek

G Hageman

Correspondence to:

Dr J van der Hoek

KCJvanderHoek@

compuserve.com

Accepted 9 February 2001
States alone, $3.7 \%$ of the general population. This number corresponds to the estimate in New Zealand, where 100000 workers $(2.7 \%$ of the general population) are exposed to solvents. ${ }^{8}$ To our knowledge, no explicit data on the incidence of CTE induced by solvents in a country or branch of industry are available.

Diagnostic criteria for CTE were introduced by the World Health Organisation (WHO) in 1985 and require "verified exposure to neurotoxic solvents; clinical picture of organic nervous system damage with typical subjective symptoms and objective findings in clinical and auxiliary examinations and more common combinations of central and peripheral nervous system abnormalities; and other organic diseases and primary psychiatric diseases reasonably well excluded". ' According to WHO, CTE can be classified in three stages: "organic affective syndrome" (type I), "mild chronic toxic encephalopathy" (type II), and "severe chronic toxic encephalopathy" (type III). Also in 1985, a workshop in Raleigh, USA, proposed a classification which recognises four stages of CTE: "symptoms only" (type 1), "sustained personality or mood change" (type 2A), "impairment of intellectual function" (type 2B), and "dementia" (type 3). ${ }^{10}$ Both classifications contain some inconsistencies. Moreover, the diagnostic criteria are not explicit and can be interpreted differently. ${ }^{11}$

Although a lot of experience must have been gained since 1985, little information is available in the literature on how the diagnostic criteria and classifications are implemented in the routine diagnostic procedures for CTE induced by solvents used in diagnostic centres in different countries. Such information is needed not only to improve the diagnostic procedure for individual patients, but also to check if comparison of data on incidence, aetiology, therapy, and preventive measures is possible. So, the objective of our study was to make an inventory of the criteria and methods used to diagnose CTE induced by solvents. We also wanted to get an impression of the number of patients referred to specialised centres and the distribution of the diagnoses made. For these reasons, we undertook a survey among international experts involved in this field.

\section{Material and methods}

We designed a concise questionnaire (five pages, 15 questions) on the routine diagnostic investigation of patients suspected of having CTE induced by solvents. The questions investigated the number of patients referred, referral, entrance criteria, disciplines involved, applied examinations, assessment of exposure, the classification used, and the diagnoses 
made; a neuropsychological protocol was asked for. Experts were selected on the basis of authorship of articles and editorials on CTE induced by solvents. More or less worldwide
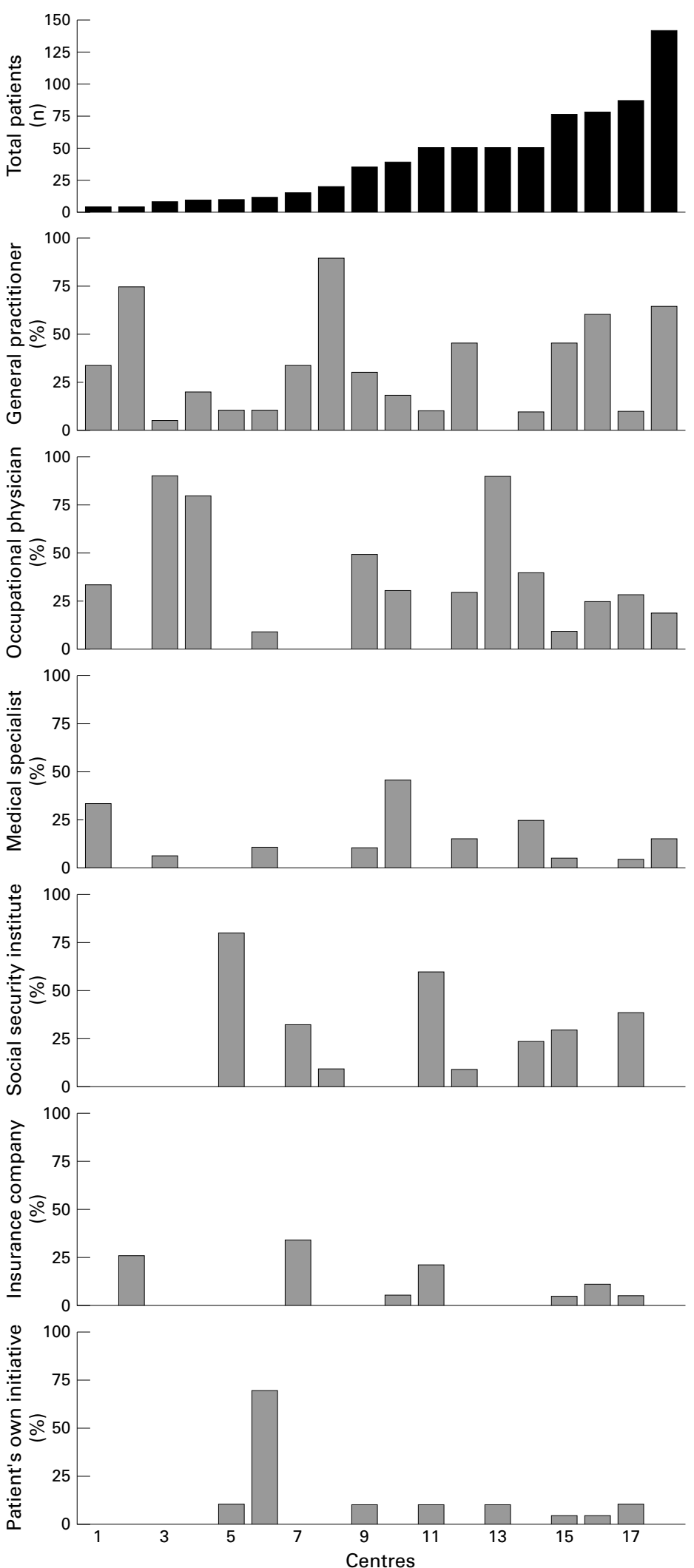

Figure 1 Number of patients suspected of CTE induced by solvents referred in 1998 to the 18 surveyed centres (top) and distribution (\%) by centre of people and institutions referring patients. coverage was intended. Twenty five questionnaires were sent out in April 1999, including five to Swedish centres because they are all involved in this field. A reminder was sent out in August 1999. All questionnaires referred to the situation in 1998 .

\section{Results}

Eighteen experts in 18 different institutes returned a completed questionnaire; three experts brought up that they and the institutes where they were employed were not or no longer involved in assessing patients for CTE. So the response was $82 \%(18 /(25-3))$ at least: among the non-respondents there may be noninvolved experts as well. Questionnaires were received from Sweden (five), United States (three), Denmark (two), and one each from Belgium, Croatia, Finland, France, Germany, the Netherlands, New Zealand, Norway, and Switzerland.

REFERRAL

The number of patients referred to the 18 centres in 1998 varied from four to 142 (mean 40 patients). Patients were mainly referred by general practitioners (GPs) or occupational physicians; their contribution, however, varied greatly (fig 1), even among the five centres in one country (Sweden), where the referral by the GP ranged from $10 \%$ to $60 \%$. Fourteen centres did not have entrance criteria with a minimum duration of exposure. The other four centres had such criteria, ranging from minimally 1 year to minimally 10 years of exposure, but exceptions were made for patients with very intensive exposure. Eight centres did not require specific symptoms or signs, five centres required cognitive or mood changes reported by the patient, and four centres required a psychometric evaluation before entry. One tertiary referral centre required that an occupational physician had already diagnosed CTE; the main function of this centre was to verify whether the disease was of occupational origin to satisfy social security requirements for financial compensation.

\section{DIAGNOSTIC PROCEDURE}

In most centres, an occupational physician or occupational specialist and a neuropsychologist examined the patients (table 1); occupational hygienists, neurologists, or psychiatrists were usually consulted when necessary. A chemist or toxicologist was seldom involved. An interview was part of the diagnostic procedure in all centres, a physical examination in 16, neurological examination in 15 , and psychological tests in 14 . In most centres, blood tests, electroencephalogram (EEG), imaging of the brain, electroneuromyography (ENMG), or evoked potentials were used on indication only (table 2). Thirteen centres used one set of core tests and two used two consecutive sets of core tests with the second set being used only when the first set showed defined impairments. Three centres did not use a set of core tests.

Ten centres sent us their neuropsychological protocol. Various tests were used to evaluate 
Table 1 Disciplines involved in the examination of patients suspected of having CTE in 18 specialised centres in 12 countries

\begin{tabular}{llcc}
\hline & Always & If indicated & Never \\
\hline (Neuro)psychologist & 11 & 7 & 0 \\
Occupational specialist & 11 & 6 & 1 \\
Occupational physician & 9 & 2 & 7 \\
Occupational hygienist & 6 & 10 & 2 \\
Neurologist & 6 & 10 & 2 \\
Toxicologist & 2 & 7 & 9 \\
Chemist & 2 & 6 & 10 \\
Psychiatrist & 1 & 11 & 5 \\
Other & Social worker (1) & Specialist in internal & \\
& Vision physiologist (1) & medicine (1) & \\
& & & \\
\hline
\end{tabular}

Table 2 Examinations used to evaluate patients suspected of having CTE in 18 specialised centres in 12 countries

\begin{tabular}{llcl}
\hline & Always & If indicated & Never \\
\hline Interview & 18 & 0 & 0 \\
Physical examination & 16 & 1 & 1 \\
Neurological examination & 15 & 3 & 0 \\
Psychological tests & 14 & 4 & 0 \\
Laboratory & 7 & 11 & 0 \\
CT/MRI & 5 & 12 & 4 \\
EEG & 5 & 9 & 4 \\
ENMG & 3 & 11 & 5 \\
Evoked potentials & 2 & SPECT/PET (1) & \\
Other & rCBF (1) & Biomonitoring $(1)$ & \\
& SPECT (1) & \multicolumn{2}{c}{} \\
\hline
\end{tabular}

$\mathrm{CT} / \mathrm{MRI}=$ computed tomography/magnetic resonance immagimg; $\mathrm{EEG}=$ electroencephalogram; ENMG=electroneuromyography; $\mathrm{RCBF}=$ regional cerebral blood flow; SPECT=single photon emission computed tomography; PET=positron emission tomography.

psychological domains; many of these tests are not internationally known. This made it impossible to interpret the differences in the distribution of the diagnoses of CTE across centres. Seven centres required at least 1 week of non-exposure before the neuropsychological tests; 11 centres had no requirements of this type. Ongoing alcoholism or a history of alcoholism was a contraindication for neuropsychological testing in six centres; two centres excluded patients who were using medication that could affect the results of neuropsychological testing, such as hypnotic drugs or tranquilisers. Other reasons for disallowing neuropsychological tests were major psychiatric or neurological disorder before exposure, and current use of marijuana or drugs. Only three

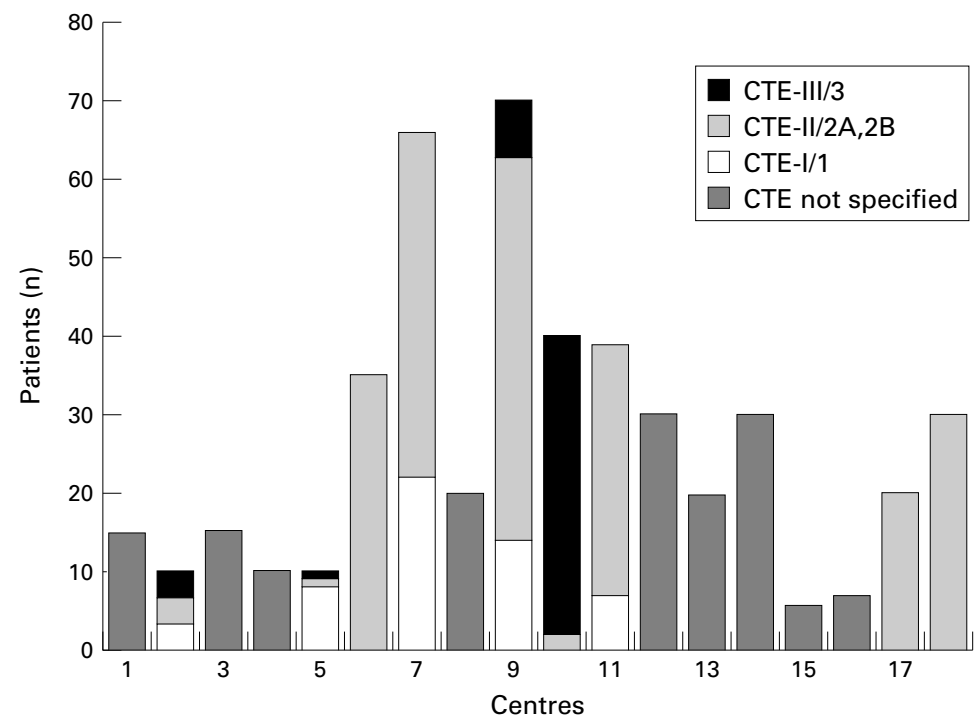

Figure 2 Distribution of final diagnoses according to WHO or Raleigh (\% of referred patients) among the centres. Centres are numbered as in figure 1. centres used tests specifically developed to detect malingering - for example, the test of memory malingering (TOMM) or the multidigit memory test (MDMT). Five centres looked for inconsistency across tests results to detect malingering, and 10 did not take the possibility of malingering into account at all.

The extent of exposure was assessed by interview in 15 centres. Five centres took measurements at the workplace as a routine, 11 centres only did so if this was indicated, and two centres never took such measurements. A few centres mentioned the use of a database (exposure matrix) or biological monitoring. Nine centres assessed earlier exposure by means of an exposure index, but these centres calculated the index in a different way. Eight centres recorded the solvents involved individually and another seven centres categorised them in groups.

CLASSIFICATION AND DIAGNOSIS

Only eight out of the 18 centres used a classification specifically developed and internationally accepted for CTE, four centres used the WHO classification, and four centres used the Raleigh classification. The other classifications comprised the 10 th revision of the international classification of diseases (ICD-10) (three times) and the diagnostic and statistical manual of mental disorders (DSM-IV) classification (once), a three stage non-international classification (twice), and a seven stage noninternational classification (once). Three centres did not use a classification system at all. In Sweden four different systems were in use. More than one diagnosis was allowed in all but one centre. In 10 centres all patients were assessed for CTE because of a claim for financial compensation; in the others this was the case for $75 \%, 40 \%, 20 \%, 10 \%, 5 \%$, and $0 \%$ of the patients. The two remaining centres responded to the question about what proportion of patients were dependent on a diagnosis of CTE for compensation "probably most of them" and "unknown".

Of the referred patients, between $6 \%$ and $70 \%$ of them were diagnosed with CTE. Of these patients $0 \%-33 \%$ were diagnosed with CTE type I/1, 5\%-100\% with type II/2, and $0 \%-95 \%$ with type III/3 according to the WHO or Raleigh classification (fig 2).

\section{Discussion}

Our survey shows that there is broad agreement that a routine diagnostic procedure for CTE induced by solvents should contain an interview, and neurological, physical, and neuropsychological examinations. However, there was a large variation in the tests used in the diagnostic procedure, and it is amazing that in four centres psychological tests were not a part of the diagnostic procedure.

Most centres assessed the extent of exposure by interview and about half assessed the working life exposure by means of an exposure index. We think that earlier exposure should be assessed by means of an exposure index and that the same method should be used by each centre to calculate the exposure index, which 
was not the case. The fact that the solvents involved were recorded individually or categorised in groups offers the chance to study possible specific effects of a solvent or a category of solvents.

Many of the neuropsychological tests in use are not internationally accepted. Clearly at least a core battery of internationally accepted and validated tests should be used. It was remarkable that malingering was often not taken into account, even though many patients enter the procedure to obtain financial compensation.

We can only assume that the diagnostic procedures used in other centres where patients suspected of CTE are diagnosed will probably not be of the same standard as those used in the centres that participated in this study. This is because our respondents are experts who are not only involved in routine diagnosis of patients but were chosen because they are also active in the field of science.

The distribution of diagnoses of CTE across the centres differed strikingly. This can be explained by several factors: the variety in indications for referral; in the assessment of the exposure; assessment of the signs and symptoms; and in the different classifications used. The use of different classifications is important: WHO type II requires only one of four selected cognitive domains (speed, attention, dexterity, and short term memory) to be impaired. By contrast, Raleigh type $2 \mathrm{~B}$ requires impairment of all three selected cognitive domains (concentration, memory, and learning capacity), although later in the workshop proceedings it is stated that "type $2 \mathrm{~B}$ most likely shows mild-to-moderate impairments in the following three functions: psychomotor functions, sustained attention/concentration, and verbal and non-verbal memory". ${ }^{10}$ Even so, the WHO and Raleigh classifications, allowing for assessment of the degree of impairment, are more informative than general classifications such as ICD-10 and DSM-IV. For instance, DSM-IV only recognises "inhalant-induced persistent dementia (292.82)" and "inhalantinduced mood disorder (292.83)", ${ }^{12}$ however the equivalent of CTE type II or 2B "inhalantinduced cognitive disorder" is not recognised. If a more general classification for a case is required-for example, for statistics or financial compensation, we suggest that such a classification should be used in combination with a specific CTE classification. The fact that the WHO or Raleigh classifications are used by only eight of the 18 centres indicates that they have their drawbacks. The most important drawback in our opinion is the lack of well described criteria. Other reasons for why the 1985 Raleigh and WHO meetings had little success may be that in those days the awareness of the potential effects of chronic solvent exposure on the CNS was limited and that the existence of the "Scandinavian solvent syndrome" was controversial. Nowadays there is little doubt that long term occupational exposure to solvents can result in a chronic encephalopathy: most nations of the European Union recognise CTE in their official list of occupational diseases. ${ }^{13}$ So the need for a univocal classification system will now be recognised more widely. For these reasons we think that activities to attain international consensus are more likely to succeed now than in 1985. Such a classification system is also necessary for international comparison of incidence, and aetiological studies of CTE and its treatment. The improved classification should contain an approach for the assessment of exposure. Also, the relevant neuropsychological domains based on internationally validated neuropsychological tests should be defined and distinct criteria for the required extent of impairment in these domains should be formulated. We think that a new CTE classification that meets these requirements would be advantageous and better than the two current classifications.

We express our gratitude to the following people for their much appreciated cooperation: Dr R Akila, Helsinki, Finland; Professor O Axelson, Linköping, Sweden; Dr L Barregård, Göteborg, sor O Axelson, Linköping, Sweden; Dr L Barregård, Göteborg,
Sweden; Professor M-A Boillat, Lausanne, Switzerland; Professor S Dally, Paris, France; Dr E W Dryson, Penrose, New Zeasor S Dally, Paris, France; Dr E W Dryson, Penrose, New Zea-
land; Dr P Gustavsson, Stockholm, Sweden; Dr H L Leira, land; Dr P Gustavsson, Stockholm, Sweden; Dr H L Leira,
Trondheim, Norway; Dr S Mikkelsen, Copenhagen, Denmark; Trondheim, Norway; Dr S Mikkelsen, Copenhagen, Denmark;
Dr L Morrow, Pittsburgh, USA; Professor P Orbæck, Lund, Sr L Morrow, Pittsburgh, USA; Professor P Orbæck, Lund, Sweden; Dr K Rasmussen, Herning, Denmark; Professor G
Triebig, Heidelberg, Germany; Dr M Viane, Leuven, Belgium; Triebig, Heidelberg, Germany; Dr M Viane, Leuven, Belgium;
Dr A Vrca, Zagreb, Croatia; Professor R F White, Boston, USA; and Dr G Wieslander, Uppsala, Sweden. We are also indebted to Professor Bassuyt for his stimulating ideas about this study.

1 Hogstedt C. Has the Scandinavian solvent syndrome controversy been solved? Scand 7 Work Environ Health 1994;20:59-64.

2 Lees-Haley PR, Williams CW. Neurotoxicity of chronic low-dose exposure to organic solvents: a skeptical review. $\mathcal{F}$ Clin Psychol 1997;53:699-712.

3 Juntunen J. Neurotoxic syndromes and occupational exposure to solvents. Environ Res 1993;60:98-111.

4 Mikkelsen S. Epidemiological update on solvent neurotoxicity. Environ Res 1997;73:101-12.

5 Morrow LA, Ryan CM, Hodgson MJ, et al. Alterations in cognitive and psychological functioning after organic solvent exposure. $\mathscr{f}$ Occup Med 1990;32:444-50.

6 White RF, Proctor SP. Solvents and neurotoxicity. Lancet 1997;349:1239-43.

7 Current Intelligence Bulletin 48. Organic solvent neurotoxicity. Cincinnati: USDHHS/PHS/CDC/NIOSH, 1987. (DHHS Publ No. 87-104.)

8 Dryson EW, Ogden JA. Chronic solvent neurotoxicity in New Zealand: notified cases between 1993 and 1997. NZ Med F 1998;111:425-7

9 World Health Organization. Chronic effects of organic solvents on the central nervous system and diagnostic criteria. Copenhagen: WHO, 1985:1-39. (Environmental Health 5.)

10 Baker EL, Seppäläinen AM. Workshop on neurobehavioral Baker EL, Seppäläinen AM. Workshop on neurobehavioral effects of solvents. Human aspects of solvent

11 Hoek JAF van der, Verberk MM, Hageman G. Criteria for solvent-induced chronic toxic encephalopathy: a systematic review. Int Arch Occup Environ Health 2000;73:362-8.

12 Quick reference to the diagnostic criteria from DSM-IV. Washington, DC: American Psychiatric Association, 1994.

13 Triebig G. Solvent related chronic encephalopathy. Editorial. Int Arch Occup Environ Health 2000;73:361. 